\title{
Empatia e afetos em estudantes do ensino superior na área da saúde
}

\section{Empathy and affectivity in students of higher education in health}

\author{
Ana Catarina Reis, Ana Salgado, Helena Martins, Andreia Magalhães, Zita Sousa, Artemisa Rocha Dores \\ Escola Superior de Saúde do Porto. P. Porto
}

\begin{abstract}
Resumo
A relevância da empatia enquanto competência clínica central nas profissões de saúde tem sido cada vez mais reconhecida. Esta pode influenciar dimensões como os resultados clínicos e satisfação dos utentes relativamente aos cuidados de saúde. Este estudo pretende analisar associações entre empatia e experiência de afetos em estudantes, do $1^{\circ}$ ano de diferentes licenciaturas da área da saúde. O estudo incluiu 241 estudantes de 12 licenciaturas, sendo a idade média 19.3 anos $(D P=2.6,17-38)$. Os estudantes preencheram a Escala de Empatia Médica de Jefferson, a PANAS e um questionário sociodemográfico. Foram encontradas associações estatisticamente significativas entre empatia e afetos $(p<.05)$.

Palavras-chave: empatia, afetos, saúde, estudantes, ensino superior.
\end{abstract}

\begin{abstract}
The importance of empathy as a core clinical competence in the context of health care professions has received increasing recognition. Empathy can influence dimensions such as clinical outcomes and patients' satisfaction. This study aims to examine the associations between empathy and affectivity in undergraduate students. The study included 241 first-year students with mean age 19.3 years $(S D=2.6,17-38)$. They responded to Jefferson Scale of Physician Empathy, PANAS and to a sociodemographic questionnaire. The results show significant associations between empathy and affectivity $(p<.05)$.

Keywords: empathy, affects, health, students, higher education.
\end{abstract}

Um dos desafios atuais da educação clínica é promover o desenvolvimento de competências afetivas e comunicacionais. Tendo como referência estudos desenvolvidos na área da Medicina (Hong et al., 2012; Medeiros, Santos, Trindade, \& Almeida, 2013; Williams et al., 2014), verifica-se que ao longo da formação há um declínio gradual da empatia (Bellini et al., 2002; Hojat et al., 2009; Newton, Barber, Clardy, Cleveland \& O'Sullivan, 2008), mas os resultados revelam-se controversos com outros estudos a evidenciarem a manutenção (Quince et al., 2011) ou mesmo aumento da empatia (Kataoka, Koide, Ochi, Hojat \& Gonnella, 2009; Magalhães et al., 2011). Estes resultados parecem revelar a importância da formação nesta área, destacando o ensino da empatia enquanto competência clínica (Tavakol, Dennick, \& Tavakol, 2012). Hulsman, Ros, Winnisbust e Bensing (1999) reforçam que o desafio da formação no século XXI no contexto da saúde é o desenvolvimento das competências de comunicação.

A empatia é considerada um atributo cognitivo e afetivo essencial dos profissionais de saúde, sendo reconhecida a sua importância na prestação de cuidados (Ogle, Bushnell, \& Caputi, 2013; Williams et al., 2014). A dimensão afetiva da empatia é a capacidade de perceber o outro e ser capaz de ver o mundo da mesma forma que este o vê (Hojat et al., 2004). A dimensão cognitiva, que pode ser alvo de treino, é a capacidade de compreender as emoções e as necessidades do outro e de comunicar esse entendimento para que o outro se sinta compreendido (Ogle et al., 2013). Estudos sugerem que os profissionais de saúde, em particular os médicos, apresentam dificuldades em lidar com os aspetos emocionais na sua prática profissional (Medeiros et al., 2013).

Os afetos positivos constituem uma componente importante para o funcionamento social adaptativo, refletindo um indicador estável da experiência emocional individual (Devlin, Zaki, Ong, \& Gruber, 2014), enquanto os afetos negativos refletem uma dimensão mais global associada ao distress $(\mathrm{Li}, \mathrm{Mo}, \mathrm{Wu}, \& \mathrm{Lau}$, 2017). Os efeitos sociais positivos resultantes da experiência emocional de afetos positivos podem ser associados à capacidade individual de empaticamente se ser capaz de compreender as emoções do outro, sendo esta uma competência relevante para desenvolver e manter relações interpessoais (Devil et al., 2014).

Os afetos positivos têm vindo a ser associados a diversos benefícios sociais, mas não é clara a sua associação com a empatia. Alguns estudos sugerem que os afetos positivos se associam a níveis mais elevados nas dimensões tomada de perspetiva e compaixão da empatia (Grühn, Rebucal, Diehl, Lumley, \& Labouvie-Vief, 2008). Por outro lado, alguns autores apontam para que nem sempre a experiência de afetos positivos seja um indicador de maiores níveis de empatia, justificando que os afetos positivos podem, em alguns casos, estar associados a resultados interpessoais desfavoráveis que podem incluir estereótipos e julgamentos sobre o comportamento dos outros (Forgas \& East, 2008). Alguns autores sugerem ainda que os episódios empáticos podem desencadear sentimentos qualitativamente distintos e, neste sentido, Batson e colaboradores (1987) desenvolveram seis estudos em que foi pedido aos participantes que avaliassem as 
reações afetivas e a intensidade associada às mesmas com base numa lista de adjetivos (alarmed, grieved, upset, worried, compassionate, tender warm, etc.). Após a análise dos resultados foi possível verificar que da lista de adjetivos fornecida, parte se relacionava com o distress (mais relacionado com a experiência de afetos negativos) e os restantes adjetivos refletiam a empatia. Neste sentido, a empatia deve ser considerada um constructo multidimensional que envolve uma resposta emocional às reações emocionais do outro (Sampaio, Camino, \& Roazzi, 2009).

Dada a escassez de estudos que relacionem a empatia com a experiência de afetos positivos e negativos junto de estudantes do ensino superior, foi objetivo deste estudo analisar estas variáveis e a sua associação em estudantes do ensino superior da área da saúde.

\section{Método}

\section{Participantes}

Participaram neste estudo 241 estudantes, do $1^{\circ}$ ano da Escola Superior de Saúde do Porto (ESS - P. Porto), dos quais $86.3 \%(\mathrm{n}=208)$ são do género feminino, com uma média de idades de 19.3 anos ( $D P=2.6,17-38$ anos). Apesar de pertencerem a 12 licenciaturas, por facilidade de análise dos dados, os participantes foram agrupados, pela equipa de investigação, em três áreas de formação distintas: Avaliação, Intervenção e Terapia (AIT Fisioterapia, Terapia Ocupacional, Terapia da Fala e Audiologia), Ciências das Radiações e Análise do Sinal Biológico (CRASB - Radioterapia, Medicina Nuclear, Radiologia, Cardiopneumologia e Neurofisiologia) e Ciências Clínico-Laboratoriais, Farmacêuticas e Saúde Pública (CCLFSP - Análises Clínicas, Farmácia, Saúde Ambiental e Anatomia Patológica, Citológica e Tanatológica).

\section{Instrumentos}

A empatia foi avaliada através da Escala de Empatia Médica de Jefferson (JSPE), versão em português para estudantes (JSPE-SPV; Magalhães, DeChamplain, Salgueira, \& Costa, 2010), que inclui 20 itens distribuídos por três dimensões: tomada de perspetiva, compaixão e capacidade para se colocar no lugar do outro. $\mathrm{O}$ instrumento apresenta-se sob o formato de resposta numa escala de tipo Likert, que varia entre 1 (discordo fortemente) e 7 (concordo fortemente). No presente estudo obtivemos os seguintes valores de alpha de Cronbach relativamente à consistencia interna das dimensões: tomada de perspetiva $\alpha=.86$, compaixão $\alpha=.70$ e capacidade para se colocar no lugar do outro $\alpha=$.71. Para avaliar os afetos foi utilizada a Positive and Negative Affect Schedule - PANAS na versão portuguesa adaptada por Galinha e Ribeiro (2005). A PANAS é constituída por 20 itens divididos em duas dimensões: dez emoções positivas e dez emoções negativas. No que se refere à consistência interna obtivemos os seguintes valores: alpha de Cronbach para a escala de afetos positivos $\alpha=.86$ e alpha de Cronbach para a escala de afetos negativos $\alpha=.85$.

\section{Materiais e Procedimento}

O trabalho apresentado é parte integrante de um estudo longitudinal aprovado pela comissão de Ética da ESS-P. Porto. Os estudantes do $1^{\mathrm{o}}$ ano foram convidados a participar através de duas formas: preenchimento em papel ou na versão informática on-line no início do $1^{\circ}$ semestre do ano letivo de 2014/2015. A participação foi voluntária e os participantes assinaram o consentimento informado, sendo garantida a confidencialidade dos dados obtidos. A análise estatística de dados foi realizada através do programa estatístico IBM SPSS (Statistical Package for Social Sciences), na versão 24.

\section{Resultados}

Diferenças na empatia e nos afetos nos estudantes do $1^{\circ}$ ano em função do género e da área de formação

Para testar a hipótese de que existem diferenças estatisticamente significativas na empatia e na experiência de afetos nos estudantes do $1^{\circ}$ ano em função do género, recorreu-se ao procedimento estatístico teste $t$ de Student para amostras independentes. Os resultados são apresentados na Tabela 1.

Tabela 1.

Diferenças na empatia e nos afetos nos estudantes do $1^{\circ}$ ano em função do género

\begin{tabular}{|c|c|c|c|c|c|c|}
\hline \multirow[t]{2}{*}{ Dimensões } & \multicolumn{2}{|c|}{$\begin{array}{l}\text { Masculino } \\
(\mathrm{n}=33)\end{array}$} & \multicolumn{2}{|c|}{$\begin{array}{c}\text { Feminino } \\
(\mathrm{n}=208)\end{array}$} & \multirow[b]{2}{*}{$t$} & \multirow[b]{2}{*}{$P$} \\
\hline & $M$ & $D P$ & $M$ & $D P$ & & \\
\hline $\mathrm{TP}(*)$ & 5.8 & .7 & 5.9 & .7 & 0.6 & n.s. \\
\hline Compaixão & 5.1 & 1.0 & 5.5 & .7 & 3.4 & .001 \\
\hline Lugar do outro & 3.8 & 1.3 & 4.3 & 1.4 & 1.9 & n.s. \\
\hline Afetos + & 32.3 & 5.3 & 30.4 & 6.8 & -1.5 & n.s. \\
\hline Afetos - & 18.8 & 6.4 & 19.3 & 6.6 & 0.4 & n.s. \\
\hline
\end{tabular}

Através da leitura da Tabela 1, é possível verificar que existem diferenças estatisticamente significativas na dimensão da empatia - compaixão - entre estudantes do género masculino $(M=5.1, D P=1.0)$ e feminino $(M=5.5$, $D P=.7, t(1,239)=3.4, p<.001)$, a favor dos estudantes do género feminino. Nas restantes dimensões não se observaram diferenças entre os grupos.

Para testar a hipótese de que existem diferenças estatisticamente significativas na empatia e na experiência de afetos nos estudantes do $1^{\circ}$ ano em função da área de formação, recorreu-se ao procedimento estatístico One-way ANOVA para comparar as três áreas de formação AIT (Fisioterapia, Terapia Ocupacional, Terapia da Fala e Audiologia), CRASB (Radioterapia, Medicina Nuclear, Radiologia, Cardiopneumologia e Neurofisiologia) e CCLFSP (Análises Clínicas, Farmácia, Saúde Ambiental e Anatomia Patológica, Citológica e Tanatológica). Os resultados são apresentados na Tabela 2. 
Tabela 2.

Diferenças na empatia e afetos nos estudantes do $1^{\circ}$ ano em função do área de Formação

\begin{tabular}{lcccccccc}
\hline Dimensões & $\begin{array}{c}\text { AIT } \\
(\mathrm{n}=83)\end{array}$ & \multicolumn{2}{c}{$\begin{array}{c}\text { CRASB } \\
(\mathrm{n}=81)\end{array}$} & \multicolumn{2}{c}{$\begin{array}{c}\text { CCLFSP } \\
(\mathrm{n}=77)\end{array}$} \\
\hline & $M$ & $D P$ & $M$ & $D P$ & $M$ & $D P$ & $F$ & $p$ \\
\hline TP $\left.{ }^{*}\right)$ & 6.1 & .6 & 5.9 & .7 & 5.7 & .9 & 2.3 & $\mathbf{. 0 1}$ \\
Compaixão & 5.6 & .7 & 5.5 & .7 & 5.4 & .9 & 1.1 & $\mathbf{. 0 3}$ \\
Lugar outro & 4.3 & 1.4 & 4.4 & 1.3 & 4.1 & 1.2 & 4.7 & n.s. \\
Afetos + & 30.2 & 6.5 & 31.7 & 6.6 & 30.1 & 6.9 & 1.5 & n.s. \\
Afetos - & 20.7 & 6.6 & 16.9 & 5.3 & 20.1 & 7.1 & 8.2 & $\mathbf{. 0 0 1}$ \\
\hline${ }^{*}$ ) TP - Tomada de perspetiva & & & & & &
\end{tabular}

Pela leitura da Tabela 2, é possível verificar que existem diferenças estatisticamente significativas em função da área de formação na dimensão tomada de perspetiva $[F(2,241)=2.3, p<.01]$ e compaixão $[F(2$, $241)=1.1, p<.05]$ da empatia e nos afetos negativos $[F(2$, $238)=8.2, \quad p<.001]$. Pela análise post-hoc Tukey, verifica-se que as diferenças estatisticamente significativas se encontram entre os estudantes de AIT (tomada de perspetiva $M=6.1, D P=.6$ e compaixão $M=5.6, D P=.7$ ) e os estudantes de CCLFSP (tomada de perspetiva $M=5.9, D P=.7$ e compaixão $M=5.4, D P=.9$ ) a favor dos estudantes de AIT em ambas as dimensões $(p<.01)$. Em relação aos afetos negativos, as diferenças estatisticamente significativas encontram-se entre os estudantes de AIT $(M=20.6, D P=6.6)$ e CRASB $(M=16.9, D P=5.3)$ a favor dos estudantes de AIT $(p<.001)$ e entre os estudantes de CRASB $(M=16.9$, $D P=5.3)$ e os estudantes de CCLFSP $(M=20.1, D P=7.1)$ a favor dos estudantes de CCLFSP $(p<.001)$.

\section{Empatia e afetos nos estudantes do $1^{\circ}$ ano}

Para analisar as associações existentes entre a empatia e a experiência de afetos dos estudantes do $1^{\circ}$ ano dos cursos de saúde da ESS, recorremos ao coeficiente de correlação momento-produto de Pearson. Analisamos as associações em função da área de formação AIT, CRASB e CCLFSP.

Tabela 3.

Coeficientes de correlação de Pearson para a empatia e afetos nos estudantes do $1^{\circ}$ ano em função da área de formação AIT

\begin{tabular}{lcc}
\hline Dimensões & Afetos + & Afetos - \\
\hline Tomada de perspetiva & $r=.36$ & $r=-.02$ \\
Compaixão & $r=.27$ & $r=.07$ \\
Lugar do outro & $r=-.05$ & $r=.13$ \\
\hline
\end{tabular}

Pela análise da Tabela 3, podemos verificar que na área de formação AIT não existem associações estatisticamente significativas entre as dimensões da empatia e a experiência de afetos positivos e negativos.

Tabela 4.

Coeficientes de correlação de Pearson para a empatia e afetos nos estudantes do $1^{\circ}$ ano em função da área de formação $C R A S B$

\begin{tabular}{lcc}
\hline Dimensões & Afetos + & Afetos - \\
\hline Tomada de perspetiva & $r=.14$ & $\boldsymbol{r}=-.31^{*}$ \\
Compaixão & $\boldsymbol{r}=\mathbf{. 3 1 *}$ & $\boldsymbol{r}=. \mathbf{2 9} *$ \\
Lugar do outro & $r=-.02$ & $r=.06$ \\
\hline$\left(^{*}\right) p<.05$ & &
\end{tabular}

Relativamente à área de formação CRASB, pela análise da Tabela 4, é possível observar que existem associações estatisticamente significativas entre a tomada de perspetiva da empatia e os afetos negativos $(r=-.31, p<.05)$. A correlação é negativa sugerindo que quanto maior for a pontuação na tomada de perspetiva, menor será a experiência de afetos negativos. No que toca à dimensão da compaixão da empatia, verificamos que se associa positivamente com os afetos positivos $(r=.31, p<.05)$ e com os afetos negativos $(r=.29, p<.05)$. As correlações encontradas sugerem que quanto maior a pontuação na compaixão, maior a experiência de afetos positivos e negativos.

Tabela 5 .

Coeficientes de correlação de Pearson para a empatia e afetos nos estudantes do $1^{\circ}$ ano em função da área de formação CCLFSP

\begin{tabular}{lcc}
\hline Dimensões & Afetos + & Afetos - \\
\hline Tomada de perspetiva & $r=.01$ & $r=-.26$ \\
Compaixão & $r=.11$ & $r=-.15$ \\
Lugar do outro & $r=-.006$ & $r=.009$ \\
\hline
\end{tabular}

Pela análise da Tabela 5, é possível verificar que na área de formação CCLFSP não foram observadas associações estatisticamente significativas entre as dimensões da empatia e a experiência de afetos positivos e negativos.

\section{Discussão}

O objetivo deste estudo foi, por um lado, analisar as diferenças nas dimensões da empatia e na experiência de afetos positivos e negativos em função do género e da área de formação de estudantes da área da saúde e, por outro, analisar as associações existentes as variáveis consideradas, em função da área de formação (AIT, CRASB e CCLFSP) dos estudantes.

Relativamente ao género, foi possível verificar que os estudantes do género feminino apresentam pontuações mais elevadas na dimensão compaixão da empatia, não se verificando diferenças entre os grupos nas restantes dimensões da empatia e na experiência de afetos positivos e negativos. Alguns estudos que também 
procuram analisar o papel do género na empatia não nos permitem retirar conclusões definitivas. Por um lado, encontram-se diferenças estatisticamente significativas em todas as dimensões da empatia em função do género, a favor do género feminino, recorrendo à mesma escala de empatia utilizada neste estudo (Ward et al., 2008). Noutro estudo não foi possível encontrar diferenças estatisticamente significativas na empatia em função do género, utilizando também a Escala de Empatia de Jefferson (Hong et al., 2014). Consensualmente, as mulheres tendem a reunir mais competências sociais e a demonstrar atitudes maternais que podem contribuir para o relacionamento interpessoal mais adequado (Ward et al., 2008), facto que pode explicar a diferença a favor das estudantes na dimensão da compaixão por nós encontrada.

No que se refere à área de formação dos estudantes, foi possível verificar que existem diferenças estatisticamente significativas nas dimensões tomada de perspetiva e compaixão da empatia e na experiência de afetos negativos. Em particular, nas dimensões da empatia são os estudantes da área AIT - Avaliação, Intervenção e Terapia - que apresentam pontuações mais elevadas em relação aos estudantes de CCLFSP Ciências Clínico-laboratoriais, Farmacêuticas e Saúde Pública. Consideramos que estes resultados se podem explicar pelos conteúdos programáticos que os estudantes de AIT abordam ao longo do seu $1^{\circ}$ ano de formação e que são orientados para a relevância da comunicação empática e do estabelecimento de uma relação terapêutica adequada entre profissional de saúde-utente que permita dar resposta às necessidades dos utentes e contribuir também para a adesão terapêutica. Outra explicação alternativa pode ser o perfil de entrada dos estudantes que escolhem cada uma das três áreas de formação, por nós criadas, ser diferente. Cada um dos agrupamentos implica um nível de relação e de competências de comunicação clínica distinto, por exemplo, superior no AIT. Este será certamente um fator considerado pelos estudantes na escolha da sua licenciatura e da sua futura profissão, determinado também pelas suas características pessoais.

Em relação à experiência de afetos negativos são os estudantes da área de formação CRASB - Ciências Radiológicas e Análise do Sinal Biológico que apresentam pontuações inferiores comparativamente às outras áreas. Consideramos que estes resultados poderão estar relacionados com um menor envolvimento esperado com o utente no futuro profissional. Esta perspetiva deve ser revista dado que segundo literatura recente estes profissionais e a qualidade da relação que estabelecem, ou não, com os utentes ser determinante de diferentes variáveis clínicas importantes como a adesão ou a repetição dos exames de rastreio dentro dos períodos recomendados (e.g., Grilo, 2012).

Analisando as correlações entre as dimensões da empatia e dos afetos positivos e negativos, verificamos que é na área de formação CRASB que existem associações estatisticamente significativas entre a tomada de perspetiva e a experiência de afetos negativos (associação negativa) e entre a compaixão e os afetos positivos e negativos (associação positiva). Não encontramos associações estatisticamente significativas nas restantes áreas de formação. Considerando outros estudos (Grilo, 2012; Tavakol et al., 2012), verifica-se que grande parte dos estudantes das áreas da saúde concordam que a empatia deve ser ensinada enquanto competência clínica e profissional, valorizando o treino desta. Este treino pode dotar os futuros profissionais de uma maior capacidade de gestão das próprias emoções, positivas ou negativas associadas à profissão, podendo explicar os resultados por nós encontrados.

Como qualquer outro estudo, também este apresenta algumas limitações que se referem ao facto da amostra não ser representativa de todos os estudantes portugueses das áreas da saúde, pelo que as conclusões devem reportar-se apenas ao universo da ESS - P. Porto. Constitui também uma limitação a recolha de dados ter sido realizada através de instrumentos de auto-relato para avaliar a empatia, não incluindo parâmetros de avaliação com base na observação.

De acordo com os resultados deste estudo, parece-nos relevante considerar que a experiência de afetos tem impacto positivo nas dimensões da empatia, pela necessidade de ser capaz de reconhecer no outro diferentes emoções (positivas ou negativas) e devolver essas mesmas emoções ao outro. Neste sentido, seria importante continuar este estudo ao longo do tempo procurando entender a influência desta e de outras variáveis no «desenvolvimento» da empatia, tais como a formação académica ou vivências académicas mais latas.

\section{Referências}

Batson, C. D. (1987). Self-report rating for empathic emotion. In N. Eisenberg \& J. Strayer (Eds.), Empathy and its development (356-360). New York: Cambridge University Press.

Bellini, L. M., Baine, M., \& Shea, J. A. (2002). Variation of mood and empathy during internship. JAMA, 287, 3143-3146.

Devlin, H., Zaki, J., Desmond, C., \& Gruber, J. (2014). Not as good as you think? Trait positive emotion is associated with increased self-reported empathy but decreased empathic performance. Plos One, 9 (10): e110470. doi:10.1371/journal.pone.0110470

Forgas, J. P., \& East, R. (2008). On being happy and gullible: mood effects on skepticism and the detection of deception. Journal of Experimental Social Psychology, 44, 1362-1367. doi:10.1016/j.jesp.2008.04.010

Galinha, I., \& Ribeiro, J. L. P. (2005). Contribuição para o estudo da versão portuguesa da Positive and Negative Affect Schedule (PANAS): II - Estudo psicométrico. Análise Psicológica, 2 (XXIII), 219-227.

Grilo, A. (2012). Ensino de competências comunicacionais em estudantes e profissionais de saúde: situação atual e perspetivas. Revista IberoAmericana de Educação Superior, 7 (3), 93-112.

Grühn, D., Rebucal, K., Diehl, M., Lumley, M., \& Labouvie-Vief, G. L. (2008). Empathy across the adult lifespain: longitudinal and experience-sampling findings. Emotion, 8, 753-765. doi:10.1037/a0014123 
Hojat, M., Vergare, M. J., Maxwellk, K., Brainard, G., Herrine, S. K., Isenberg, G. A...Gonella, J. (2009). The devil is in the third year: a longitudinal study of erosion of empathy in medical school. Acad Med, 84 (9), 1182-1191. doi:10.1097/ACM.0b013e3181b17e55

Hojat, M., Mangione, S., Nasca, T., Rattner, S., Erdmann, J., Gonnella, J., \& Magee, M. (2004). An empirical study of decline in empathy in medical school. Medical Education, 38, 934-941.

Hong, M., Lee, W. H., Park, J. H., Yoon, T., Moon, D. S., Lee, S. M., \& Bahn, G. H. (2012). Changes of empathy in medical school students: 1-year follow up study. BMC Medical Education, 12, 122. doi:10.1186/1472-6920-12-122

Hulsman, R., Ros, W., Winnubst, J., Bensing, J. (1999). Teaching clinically experienced physician's communication skills a review of evaluation studies. Med Educ, 33, 655-68.

Kataoka, H. U., Koide, N., Ochi, K., Hojat, M., \& Gonnella, J. S. (2009). Measurement of empathy among Japanese medical students: Psychometrics and score differences by gender and level of medical education. Acad Med, 84, 1192-1197.

Magalhães, E., DeChamplain, A., Salgueira, A., \& Costa, M. J. (2010). Empatia Médica: Adaptação e Validação de uma Escala para Estudantes de Medicina. Atas do VIII Simpósio de Investigação em Psicologia (77-89). Braga.

Medeiros, N., Santos, T., Trindade, E. V., \& Almeida, K. (2013). Avaliação do desenvolvimento de competências afetivas e empáticas do futuro médico. Revista Brasileira de EducaçãoMédica, 37(4),515-525. https://dx.doi.org/1 0.1590/S0100-55022013000400007

Newton, B. W., Barber, L., Clardy, J., Cleveland, E., \& O'Sullivan, P. (2008). Is there hardening of the heart during medical school? Acad Med, 83, 244-249.

Ogle, J., Bushnell, J. A., \& Caputi, P. (2013). Empathy is related to clinical competence in medical care. Medical Education, 47, 824-831. doi: 10.1111/medu.12232

Sampaio, L., Camino, C., \& Roazzi, A. (2009). Revisão de aspectos conceituais, teóricos e metodológicos da empatia. Psicologia, Ciência e Profissão, 29 (2), 212-227.

Tavakol, S., Dennick, R., \& Tavakol, M. (2012). Medical students' understanding of empathy: a phenomenological study. Medical Education, 46, 306-316.

Ward, J., Schall, M., Sullivan, J., Bowen, M., Erdmann, J., \& Hojat, M. (2008). The Jefferson Sclae of Attitudes toward Physician-Nurse Collaboration: A study with undergraduate nursing students. Journal of Interprofessional Care, 22 (4), 375-386. doi:10.1080/1351820802190533

Williams, B., Brown, T., Boyle, M., McKenna, L., Palermo, C., \& Etherington, J. (2014). Levels of empathy in undergraduate emergency health, nursing and midwifery students: a longitudinal study. Advances in Medical Education and Practice, 5, 229-306. https://doi.org/10.2147/AMEP.S66681

\section{Agradecimentos}

Agradecemos a todos os estudantes que aceitaram colaborar neste estudo, permitindo a sua concretização e conhecimento nesta área. 